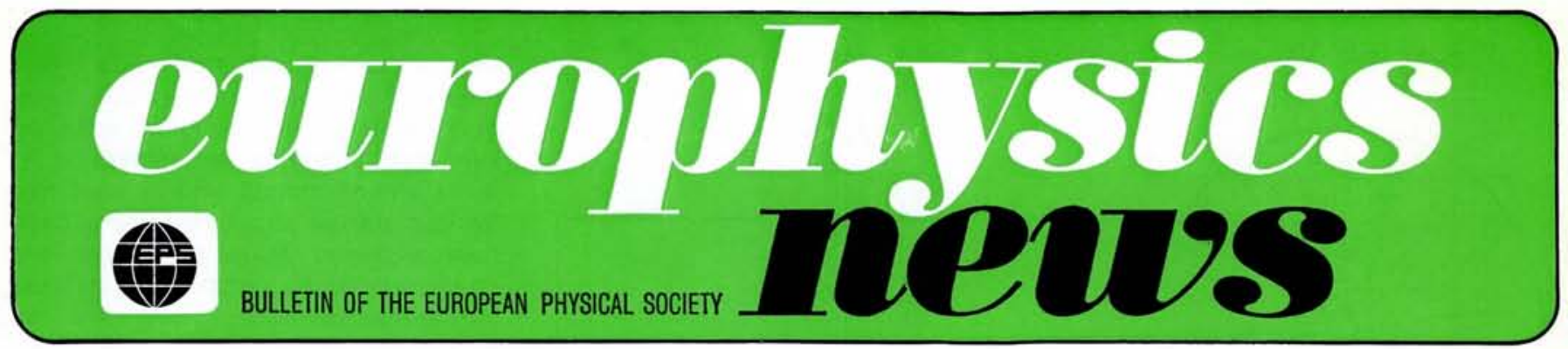

\title{
Neutron Beam Research at the High Flux Reactor of the Institute Max von Laue-Paul Langevin
}

\author{
R. L. Mössbauer, Grenoble
}

The convention for the creation of the Institute Max von Laue - Paul Langevin (ILL) was signed in January 1967 by France and the Federal Republic of Germany, with the aim to construct and operate a high flux neutron beam reactor and its associated instrumentation. On 1 January 1973, the United Kingdom has joined the Institute as third full member, contributing a fair share to the past investment.

The ILL operates under the jurisdiction of a Steering Committee, with a Scientific Council advising the Director. The Institute is largely operating as a user's facility, with about $70 \%$ of its neutron beam time being reserved for experiments proposed by external laboratories. The majority of these experiments originate in the three member countries, with scientists from other countries frequently collaborating. The Scientific Council has set up subcommittees, which specialize in specific domains and which meet twice a year in order to review the submitted experimental propositions. In conformity with its main purpose as user's facility, the Institute has been organized such as to render as easy as possible the use of its neutron beams and of its instruments, providing advice and assistance in the running of experiments, if necessary.

In general, all those facilities are made available by the ILL, which are of general use in research with neutron beams, including spectrometers, data acquisition systems, detectors, shielding, cryostats, furnaces, pressure facilities, while the users are supposed to provide those components which are specific to a particular experiment, especially the samples to be studied.

A total of 360 persons are presently employed, including some 120 scientists. An operation with this relatively small staff is only possible by extensive use of facilities of the adjacent French Research Centers, as well as by close collaboration with other Research Centers in the three countries.

\section{The High Flux Reactor (HFR)}

and its Special Installations

The HFR at Grenoble employs a single fuel element with $8.6 \mathrm{~kg}$ of ${ }^{235} \mathrm{U}$, usuable for 42 days of continuous operation. $40 \mathrm{t}$ of heavy water are used for the thermalization of the neutrons and for the primary cooling system. The HFR at Grenoble generates a total power of $57 \mathrm{MW}$ with a peak power density of $3 \mathrm{~kW} / \mathrm{cm}^{2}$, yielding a maximum flux of $1.2 \times 10^{15}$ thermal neutrons $/ \mathrm{cm}^{2} / \mathrm{sec}$.

The reactor distinguishes itself by three installations:

1. A cold source consisting of $25 \mathrm{I}$ of liquid deuterium boiling at $25 \mathrm{~K}$

2. A hot source consisting of a graphite block at $2200 \mathrm{~K}$

3. An extensive system of neutron guides.

The cold and hot sources respectively displace at their location the maxima of the neutron flux towards

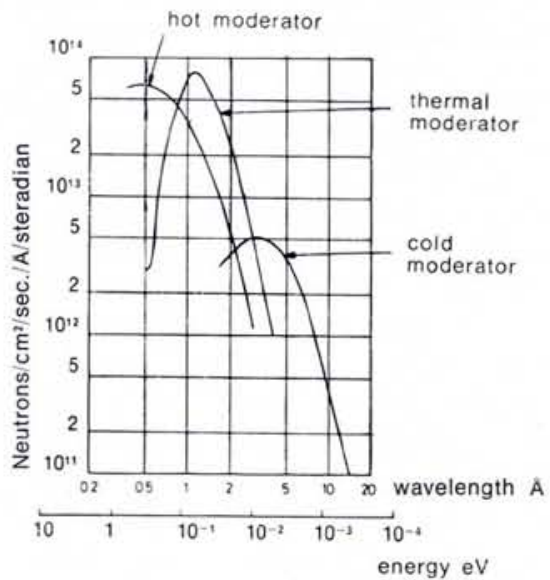

Fig. 1 Spectral neutron brightness for different moderators at the High Flux Reactor. energies somewhat below or above the usual thermal neutron energies compare Fig. 1. Many new experimental approaches became feasible due to this increased spectral range. The neutron guides are used to transport neutrons without the usual quadratic loss in intensity with distance. They employ the principle of total reflection which applies to neutrons incident on a surface below a critical glancing angle $\alpha$. For Nickel, which is the reflecting material of the neutron guides installed at the ILL, one has $\alpha \cong 0.1 \lambda$, where $\alpha$ is measured in degrees and $\lambda$ in Ångström. Neutrons of sufficiently long wavelengths, specifically those coming from the cold source, therefore become totally reflected within angular ranges, as they are typical for neutron spectrometer collimations. The ILL neutron guides produce intensity losses of the order of only $1 \%$ per $\mathrm{m}$ of guide length. They made it possible to install in regions far away from the reactor a much larger number of instruments than could be placed in its immediate vicinity, thereby increasing the measuring facilities by nearly a factor of three. The neutron guides, besides, yield a particular low background of fast neutrons and of $\gamma$-rays.

\section{Instrumentation} and Research Program

The neutron research program at the ILL comprises studies in the domains of solid state physics, che-

\section{Contents}

Neutron Beam Research at the Institute Laue-Langevin Technology arising from

High Energy Physics

Society News 


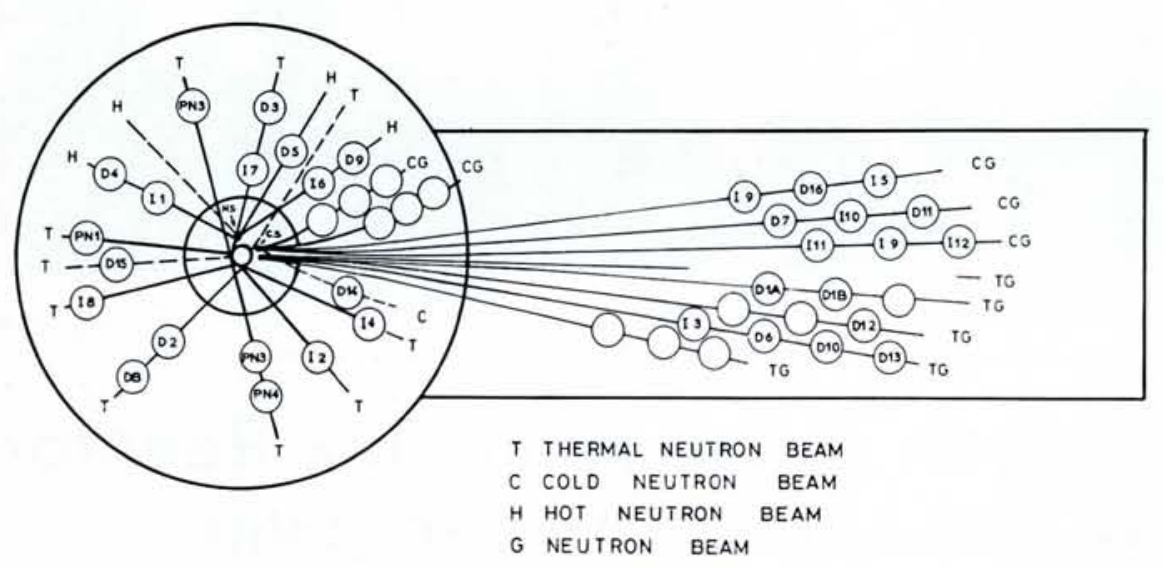

Fig. 2 Instrument arrangement around the reactor and at the neutron guides. $C, T$, and $H$ specify neutrons arriving from cold, thermal and hot moderators, respectively. $G$ designates a neutron guide, $D$ and IN refer, respectively, to diffraction and inelastic scattering equipment. PN are nuclear physics spectrometers.

mistry, biology and nuclear physics. The Institute, in fact, is subdivided into 8 colleges, concerned with theoretical physics, nuclear physics, elementary excitations (coherent inelastic scattering), crystal structures (diffraction studies), imperfect crystals, liquids and amorphous systems, physical chemistry, and biology. In 1973, some 200 experiments have been carried out, ranging in measuring time between a few hours up to one year. A survey of the different instruments which have been installed on the various beam holes around the reactor and on the neutron guides, using either cold, thermal or hot neutrons is given in Fig. 2.

The experimental research program receives substantial support from the theoretical group, which contributes new ideas and help in the interpretation of measurements. This group, besides, carries out an active research program of its own, covering a wide range of interest far beyond the realm of neutron physics.

\section{Neutron Diffraction}

The ILL carries a substantial neutron diffraction program. In this domain neutrons often serve as a supplementary technique to $\mathrm{X}$-ray scattering and prove superior in many diffraction studies, due to the unsystematic variation of their scattering cross sections with atomic or isotopic number, due to the ease with which light elements, in particular hydrogen, can be observed and also due to the possibility of magnetic scattering studies. The various two circle and four circle diffractometers of the ILL are intensively used to solve cristallographic and magnetic structure problems on powders and on single crystals. We cite as examples measurements of bond lengths in various systems, studies of hydrogen bridges, and magnetic structure studies on solid solutions, actinide compounds and on systems which are ordered in one or two dimensions. Particular problems arise in the structure analysis of crystals with very large elementary cells, as they are typical for macromolecular systems such as proteins. The usual Fourier inversion methods allow to deduce the electronic or nuclear position density in the elementary cell if the moduli and phases of the scattering amplitudes are known for a sufficiently large number of Bragg reflections. For small elementary cells direct methods are available which yield the elementary cell structure on the basis of diffraction intensity measurements alone, where the phase information gets lost. In the case of large elementary cells, however, a knowledge of the phases is indispensable and a special four circle single crystal diffractometer has been set up which will be engaged in such studies. The diffraction measurements will employ proteins which have been doped in well known positions with nuclear isotopes exhibiting thermal neutron resonances and therefore providing anomalously large scattering amplitudes. We cite the examples of ${ }^{149} \mathrm{Sm}$ with a resonance at $0.92 \AA$ and of ${ }^{157} \mathrm{Gd}$ with a resonance at $1.6 \AA$. Diffraction studies are also performed on liquids where one determines the angular dependence of the differential coherent scattering cross section. A Fourier transform from momentum space to ordinary space yields then in principle the static pair distribution function. Neutron scattering offers the possibility of changing the isotopic composition which permits direct measurements of partial pair distribution functions in two- or multi-component liquids. Experiments have been performed on binary liquid alloys, on molten salts, on saturated solutions, on molecular liquids and on metalammonia solutions. Various partial structure factors have been deduced. Molecular liquids are characterized by certain well defined interatomic distances which give rise to sharp peaks in some partial pair distribution functions and therefore to long range oscillations in momentum space, i.e. in the differential scattering cross section. Consequently, measurements must be taken up to large momentum transfers to get accurately transformable data. High energies are likewise desired in order to reduce inelastic scattering contributions. These demands are met by a special diffractometer at the ILL, which operates with neutrons from the hot source and allows for large momentum transfers.

The intensity problem in single crystal diffraction studies becomes more difficult with increasing elementary cell size, as a consequence of the growing tendency for phase randomisation together with the necessity to measure larger numbers of reflections and to apply more stringent collimation conditions. It therefore appears natural to employ multidetector systems which permit simultaneous measurements of a large number of reflections. Several such systems are either already in use or in the development stage. One system uses 100 discrete $\mathrm{BF}_{3}$-counters which are mounted on the inside of a sphere of $1.7 \mathrm{~m}$ diameter, all viewing the centrally positioned single crystal specimen. The computer controlled counters can be shifted to the individual Bragg reflections which appear in characteristic directions for each particular orientation of the single crystal studied. A bent monochromator crystal focusses a wavelength band onto the single crystal, with different wavelengths arriving from different directions, giving rise to many and well separated reflections. The same principle of a simultaneous generation of many reflections, but using photographic registration methods, is likewise being actively pursued at the ILL.

\section{Small Angle Scattering}

Another multicounter system with 4096 cells, of $1 \mathrm{~cm}^{2}$ detection area each, is associated with the small angle scattering instrument of the ILL. This instrument has a variety of applications. It gives scale information on samples with internal periodicities that are large compared to typical interatomic distances in condensed matter. This 
is, e.g., the case with certain minerals as well as with large polymer chains and with macromolecular crystals of biological interest, such as proteins. The instrument is likewise used in studies of the size and distribution of large heterogeneities, such as of agglomerates of impurities in alloys or of colloidal systems, thereby giving rise to many metallurgical and biological applications. We mention specifically studies on composite biological systems, such as membranes, viruses, ribosomes, chromatines, fibruous materials. One often makes extensive use of the fact that $H$ and $D$ as well as $\mathrm{H}_{2} \mathrm{O}$ and $\mathrm{D}_{2} \mathrm{O}$ have coherent scattering amplitudes of opposite sign, thus permitting to vary or even cancel the scattering contributions from solvent material, crystal water or from certain subgroups. We finally mention that disoriented magnetization domains give rise to mutiple refraction, with the initiated small angle scattering yielding information on domain sizes. The resolution in all these applications grows with increasing wavelength, and with better beam collimation, whence the small angle scattering spectometer of the ILL employs a cold neutron guide and distances up to $40 \mathrm{~m}$ each between monochromator and sample and sample and detector.

\section{Inelastic Scattering to}

\section{Study Dynamical Behaviour}

The main advantages of neutron scattering techniques become apparent in the regime of inelastic scattering, where neutrons can largely cover the whole range of energy and momentum transfers which occur with matter in the condensed state. This makes neutrons ideally suited for studying the dynamical behaviour of solids and liquids. By contrast, photon scattering processes either lack in energy resolution as is the case with $X$-rays or suffer from momentum limitations as is the case with Raman, infrared, Laser, NMR, ultrasonic or dielectric measurements. Neutron inelastic scattering experiments are very demanding on intensity and therefore are particularly well suited for a high flux reactor. Such experiments involve measurements of the intensity of the scattered neutrons as function of energy and scattering angle, or rather as function of the transfers of

energy $\left(E_{f}-E_{i}\right)$ and momentum $\left(\vec{k}_{f}-\vec{k}_{i}\right)$ which occur in the studied probe during the scattering process.

Coherent inelastic scattering reveals the presence of cooperative motions in matter. Such studies are being pursued at the ILL primarily by means of several triple axis spectrometers of varying resolution, which allow for independent control of the monochromator, analyser and specimen crystal orientations. We cite as examples studies of phonons and magnons in systems with one- or two-dimensional electric or magnetic ordering properties, a first measurement of a transversal acoustic phonon branch in a single crystal of a polymer, studies of critical scattering in the vicinity of phase transitions, measurements of phonon line widths, studies of intermolecular vibrations in molecular crystals.

Incoherent inelastic scattering, by contrast, yields information on the dynamical behaviour of individual particles. It is particularly well suited for the study of non-periodic motions, such as random rotations of molecules in solids, impurity diffusion in solids, translatory or rotary molecular motions in liquids, side-group motions in polymer chains, diffusion of chain segments in dilute polymers. Hydrogen, due to its large incoherent cross section, is particularly well suited for this kind of investigations and one may readily study such questions as the diffusion of hydrogen in metals or in certain molecular subgroups, which can be distinguished by replacing the hydrogen contents of other subgroups by deuterium. The whole realm of such studies is being carried out at the ILL, largely by means of time of flight neutron spectrometers, where the incident neutrons are monochromatized and pulsed by mechanical means such as rotating crystals or slit systems and where an energy analysis in various directions is then achieved by means of time of flight techniques. Some of theses instruments achieve particular high resolution in energy. A multichopper system has been designed which, with this purpose in mind, is to operate at $20000 \mathrm{rpm}$., employing slow neutrons and using a distance of $6 \mathrm{~m}$ between the energy selecting discs, an option arising out of the use of intensity saving neutron guides between the discs. Another instrument of the triple axis type eliminates one of the prime sources for the loss in energy resolution, i.e. the wavelength spread in a beam reflected from a monochromator crystal, which is caused by the finite divergence in the incident polychromatic spectrum. This spread vanishes for a scattering angle of $180^{\circ}$. Backscattering, therefore, is used in both the monochromator and analyser crystals of the instrument, while simultaneously employing Doppler shift techniques to compensate for energy differences between inci-
SUPERFLUID HYDRODYNAMICS by S. J. PUTTERMAN,
University of California

(North-Holland Series in Low Temperature Physics, Volume 3)

A central reason for interest in superfluids is that they display quantum effects on the macroscopic level and hydrodynamics on the microscopic level. The experiments which motivate this point of view and the resulting superfluid hydrodynamics theory are discussed in detail.

The minimum underlying assumptions that are required in order to derive the phenomenological theory from macroscopic first principles are discussed. Solutions of the superfluid Helium hydrodynamics include: 1st, 2nd, 3rd, 4th sound, shock waves, superfluid transverse sound, thermal and superfluid vortices, the Venturi tube, thermal superconductivity, and thermal fluctuations:

Other topics include the viscosity paradox, the rotation paradox, quantization of circulation, and the motion and drag on quantized vortices.

In addition the problem of the critical velocities and possible shortcomings of the superfluid hydrodynamics are discussed.

The magnetohydrodynamics of superconductors is presented along with its relationship to superfluid Helium. Topics include: Meissner effect quantization of the fluxoid, mixed state at type II superconductivity, superconducting Bernouilli effects. josephson effects, thermoelectric effects and the superconducting bulk viscosity.

The thermohydrodynamics of the condensed ideal Bose gas and its ielationship to the hydrodynamics of superfluid Helium is elucidated.

The approach in this book is historical wherever possible.

Remaining puzzles are emphasized.

1974, approx. 450 pages.

Dfl. 125.00 (approx. US\$ 45.50)

\section{North-Holland Publishing Co. P.O. Box 211 Amsterdam The Netherlands}

Distributor in the U.S.A. and Canada:
American Elsevier Publishing Company 52 Vanderbilt Avenue New York, N.Y. 10017 
dent and scattered neutrons due to inelastic scattering in the sample under study. Neutron resolutions smaller than $10^{-6} \mathrm{eV}$ lead to time scales in the motion of particles as long as $10^{-9} \mathrm{sec}$, which now can be investigated.

\section{Polarized Neutrons}

The use of polarized neutrons, although again at the expense of intensity, greatly adds to the experimental versatility. It becomes possible to distinguish between scattering processes with or without spin-flip of the neutron, if one employs neutron spin-polarization before and spin-analysation after the sample. Such studies, in fact, are most useful to separate different components in the scattered beam, e.g. coherent from spin-incoherent nuclear scattering in solids and liquids, incoherent scattering from randomly distributed isotopes and from randomly oriented nuclear moments, paramagnetic scattering from other types of incoherent scattering, magnon from phonon scattering in antiferromagnets. The ILL operates two instruments equipped with spin analysation facilities, one using a crystal spin-analyser in elastic scattering studies, the other one combining an energy analysis by time of flight techniques with a. spin analysis by transmission measurements through a dynamically polarized proton filter.

\section{Experiments in Nuclear Physics}

Several neutron beams are used for experiments in the domain of nuclear physics. The nuclear fission process is being studied in detail by means of a huge mass spectrometer. This instrument separates on-line the heavy fission fragments which are ejected by a foil of fissionable material exposed to a very high neutron flux. The available high resolution serves to measure the fission yield for individual nuclear masses and charges as a function of the kinetic energy of the fission fragments. A fairly complete parameter set can be obtained, especially in magic regions, if one works in the limit of high kinetic energy, where prompt neutron emission can be excluded. Odd-even effects should then be clearly visible. Investigations of the primary nuclear charge distributions are of particular interest, since previous studies gave contradictory results with respect to the influence of closed shells. Other investigations with the mass separator include studies of delayed neutron and delayed $\gamma$-emission as well as studies of $\beta$-decay chains, such as measurements of $Q$-values far off $\beta$

the beta stability curve by means of a continuous tape transport for the separated fission products.

The study of nuclear level schemes is carried out by means of several high resolution bent-crystal $\gamma$-spectrometers in combination with an anticompton and a pair-spectrometer and by a high-resolution conversion electron spectrometer. All these spectrometers view on-line sources which are mounted inside the reactor in regions of very high flux, thus extending the measuring range to isotopes with much smaller cross sections than could previously be investigated.

\section{Outlook}

The basic properties of the neutron, because of its elementary nature, continue to be of fundamental interest for our understanding of the natural for-

\section{Application for the Use of ILL Facilities}

Deadlines for the acceptance of experimental proposals are 15 February and 31 August.

All research proposals should be addressed to :

Office of the Scientific Secretary Institut Laue-Langevin

\section{B.P. 156 Centre de tri}

F-38042 Grenoble Cedex

Tel. (76) 974111 - ext. 8244

This office also provides appropriate forms for experimental proposals, information on instruments, publication lists, annual reports, annual abstracts of performed experiments.

ces. Sophisticated experiments have been devised in the past to measure various properties of the neutron, including the behaviour of its wavefunction under symmetry operations in space or in time. More such experiments are in preparation or in progress at the ILL. A search for the dipole moment of the neutron is being pursued by looking for a change in the Larmor precession frequency of a neutron in a magnetic field, if an electric field is added in parallel. This search for a dipole moment is intimately connected with the problem of the validity of time reversal invariance, which became seriously questionned after the discovery of certain forbidden modes in the decay of neutral K-mesons. A direct search for a violation of time reversal invariance has also been performed at the ILL by a study of the decay products of spin polarized neutrons, with the result of a substantial reduction of previous observation limits. New searches for a charge of the neutron and a remeasurement of its magnetic dipole moment are in progress. Se- veral searches for parity violation in nuclear forces are likewise in preparation.

The active research program in progress around the various instruments is supplemented by a number of research projects which aim at the introduction of new techniques into the field of neutron spectroscopy. Amongst those, we mention specifically the development of efficient techniques for the spin-polarization of polychromatic neutron beams, such as magnetic hexapol systems and magnetic neutron guides as well as of devices capable of electronically producing monochromatic neutron pulses by periodically generating proper magnetic scattering conditions. Other programs being pursued are the development of a new high resolution spectrometer for inelastic scattering, which measures the accumulated difference in the Larmor precession angle which a neutron experiences while travelling through magnetic fields before and after the sample. Such a difference reflects the velocity change which a neutron undergoes in an inelastic scattering process. This instrument aims at resolutions in energy as low as $10^{-9} \mathrm{eV}$. Further studies aim at the creation of a source of ultracold neutrons with wavelengths in the range of several hundred Ångström and the development of neutron interferometer techniques. Last not least, we mention the existence of an extensive program for the development and examination of monochromator crystals. This program comprises e.g. gamma-ray diffraction studies of mosaic-structures and of their homogeneities, the development of methods for controlling mosaic-structures, the development of crystals with focussing properties, both with fixed and with variable focussing lengths.

It has been quite impossible in this article to give proper credit to the many individuals inside and outside of the ILL, who have contributed to the creation of the elaborate instrumentation and who have participated in the accomplishment of the extended research program. The scale and intensity of the international collaboration which is in effect at the Institute, the diversity and scope of its scientific program, the versatile composition and the outstanding features of many of its instruments, with some 40 experiments which can be simultaneously carried out on the various beams, this all classifies the ILL as a rather unique European Research Center whose attractiveness reflects itself in the ever growing number of users and of visitors from all over the world. 